\title{
APOYO ADAPTATIVO BASADO EN IMS-LD Y ESTRATEGIAS PSICO- EDUCATIVAS PARA LA FAMILIARIZACIÓN DE ESTUDIANTES CON DISCAPACIDAD CON SU ENTORNO VIRTUAL DE APRENDIZAJE
}

\author{
(ADAPTATIVE SUPPORT BASED ON IMS-LD AND PSYCHOEDUCATIONAL STRATEGIES FOR \\ STUDENTS FAMILIARIZATION WITH THE VIRTUAL LEARNING ENVIROMENT)
}

Elena del Campo

Mar Saneiro

Psychobiotecnomedicine Research Group. Evolution and Educational Psychology

Dept., UNED (España)

Manuel F. Montecelo

Emmanuelle Raffenne

Alejandro Rodriguez-Ascaso

Olga C. Santos

Jesus G. Boticario

Grupo de Investigación aDeNu, Departamento de Inteligencia Artificial, ETSI

Informática, UNED (España)

\section{RESUMEN}

Este trabajo se enmarca en las investigaciones realizadas en los proyectos EU4ALL (IST-2006034778) y A2UN@ (TIN2008-06862-Co4-01/TSI y TIN2008-06862-Co4-00/TSI) centrados en el soporte a instituciones de Educación Superior en las actividades que deben llevarse a cabo para garantizar la participación de alumnos con discapacidad. Tanto la accesibilidad como la usabilidad de los contenidos y actividades académicas a desarrollar en el ámbito de los estudios superiores son temas abordados en las etapas de la investigación. Desde una perspectiva inclusiva que considere las necesidades específicas del estudiante se han desarrollado diseños instruccionales que tienen en cuenta distintos tipos de estudiantes, que presentan diferentes necesidades de apoyo, utilizando estándares tecnológicos educativos como la especificación IMS-LD (Instructional Management System- Learning Design) e implementándola a través del soporte proporcionado por la plataforma aprendizaje dotLRN. Dichos diseños se convierten entonces en unidades de aprendizaje ejecutadas por la plataforma en las que se adaptan recursos, contenidos y flujos de aprendizaje a las características y necesidades específicas de los estudiantes con discapacidad y/o dificultades de aprendizaje en Enseñanza Superior (dislexias, deficientes auditivos, problemas atencionales, etc.). En el diseño de actividades, contenidos y recursos se integran estrategias de aprendizaje dirigidas a mejorar las competencias del usuario para conseguir atender sus necesidades de forma interactiva, a través de distintos caminos de aprendizaje prediseñados. 


\author{
E. Campo; M. Saneiro; M. Montecelo; E. Raffenne; A. Rodriguez; O. Santos; J. Boticario \\ Apoyo AdAPTATIVo BASADO EN IMS-LD y ESTRATEGIAS PSICO-EDUCATIVAS PARA LA FAMILIARIZACión...
}

Así, la atención a la diversidad se integra con la ofrecida al resto de sus compañeros, consiguiendo resultados idóneos que pueden ser reutilizados en diferentes contextos y cursos.

Palabras clave: diseños instruccionales, estrategias de aprendizaje, adaptación, discapacidad, diversidad funcional.

\begin{abstract}
This work is part of the research in EU4ALL project (IST-2006-034778) and A2UN @ (TIN200806862-C04-01/TSI and TIN2008-06862-C04-00/TSI) focused on supporting institutions Higher Education in the activities to be carried out to ensure the participation of students with disabilities. Both accessibility and usability of content and academic activities to develop in the field of higher education, are issues addressed in the research stages. From an inclusive perspective that considers the needs of the student instructional designs have been developed taking into account different types of students who have different support needs, using educational technology standards such as IMS-LD (Learning Management System-Instructional Design) and implementing it through the support provided by the platform dotLRN learning. These designs are then converted into learning units implemented by the platform to suit the resources, content and learning flow characteristics and needs of students with disabilities and / or learning disabilities in Higher Education (dyslexia, hearing impaired, attentional problems, etc.). In the design of activities, content and resources are integrated learning strategies aimed at improving the skills of the user to get their needs in an interactive way, through various pre-designed learning paths. Thus, attention to diversity is integrated with that offered to other peers, achieving ideal results that can be reused in different contexts and courses.
\end{abstract}

Keywords: instructional design, learning strategies, adaptation, disability, functional diversity.

El apoyo psicoeducativo a estudiantes con diversidad funcional tanto a nivel físico como cognitivo es una necesidad constante a lo largo de las diferentes etapas educativas independientemente de la metodología educativa elegida. Cuando el apoyo es facilitado a través de un entorno virtual de aprendizaje, presenta características diferentes a las que encontramos cuando se produce en un entorno tradicional. La efectividad y la eficacia del mismo dependen de diferentes factores que pueden afectar el rendimiento y los resultados alcanzados por el estudiante en un entorno de estas características. Existen diversas investigaciones, como la realizada por Hasarim (2000), en la que se analiza la implicación de factores de naturaleza psicológica en el aprendizaje virtual, algunas de ellas centradas en la ansiedad relacionada con la comunicación, el manejo del tiempo, el sentimiento de estar compitiendo con otros en lugar de estar compartiendo un entorno colaborativo, etc. Swan (2000) estudia la motivación como un factor facilitador para contribuir eficazmente en una tarea de 
E. Campo; M. Saneiro; M. Montecelo; E. Raffenne; A. Rodriguez; O. Santos; J. Boticario APOYO AdAPTATIVo BASAdo EN IMS-LD y ESTRATEGIAS PSICO-EDUCATIVAS PARA LA FAMILIARIZACión...

naturaleza colaborativa, la motivación estaría además relacionada con la experiencia de interacción del usuario con el sistema, la facilidad de uso del mismo, la claridad con la que están definidos sus espacios, etc.

Es además esencial considerar que el aprendizaje en personas adultas presenta características propias que le diferencian del proceso experimentado por jóvenes y niños, ya que está afectado tanto por sus experiencias pasadas como por sus expectativas futuras (Cercone, 2008). A este fenómeno se le añade además un extra, que es la condición de diversidad funcional que presentan algunos de estos sujetos, ya que condiciones derivadas de la misma ocasionan que éstos puedan tener particularidades que afectan a las competencias necesarias para alcanzar un aprendizaje óptimo, resultándoles los contenidos y recursos inaccesibles tanto desde el punto vista físico como cognitivo.

La adaptación del entorno de aprendizaje y sus diferentes recursos y servicios a las necesidades y preferencias del usuario, requiere de la consideración por parte de los diseñadores y creadores de los contenidos educativos de: I) aquellos aspectos psicológicos del aprendizaje propios del individuo (motivación, ansiedad, autoconfianza, memoria, razonamiento, habilidades sociales y comunicativas, conocimientos previos, estilo de aprendizaje, etc.) que pueden impactar en su grado de desempeño (Martín, 2007), y II) las características propias de un entorno virtual, reseñadas por varios autores como Logan (1994), que analiza lo que denomina "usabilidad emocional", o Hofmeester (1996), que introduce el concepto de "sensualidad de la interfaz del usuario". Estos factores están íntimamente relacionados con la interacción entre el usuario y la tecnología y ponen de manifiesto la necesidad de que ésta se adapte a las necesidades y preferencias de los usuarios para conseguir un mayor grado de motivación, rendimiento y satisfacción en el aprendizaje a través de un entorno de estas características.

\section{OBJETIVO}

El grupo de investigación aDeNu de la UNED, al que pertenecen los autores de este artículo, viene desarrollando desde hace tiempo a través de diversos proyectos de investigación, diversas actuaciones en materia de apoyo a estudiantes con discapacidad en Educación Superior. Los resultados mostrados en este artículo, se engloban dentro de una de las actividades realizadas en el ámbito de los proyectos EU4ALL y A2UN@, financiados por la Unión Europea y el Ministerio de Ciencia y Tecnología respectivamente, centrados en el desarrollo de herramientas TIC accesibles y adaptativas que consideran las necesidades de todos los estudiantes. 
E. Campo; M. Saneiro; M. Montecelo; E. Raffenne; A. Rodriguez; O. Santos; J. Boticario Apoyo AdAPTATIVo BASADO EN IMS-LD y ESTRATEGIAS PSICO-EDUCATIVAS PARA LA FAMILIARIZACión...

El objetivo fundamental de ambos proyectos es la creación de una arquitectura basada en estándares que den soporte a un entorno de aprendizaje, adaptado a la necesidades y preferencias de los usuarios con discapacidad que participan en el proceso de enseñanza y aprendizaje, que elimine o minimice las limitaciones que a menudo existen en el acceso a la arquitectura y al propio entorno de aprendizaje en el que se integran los contenidos didácticos, adaptando y mejorando su metodología de aprendizaje a través de un uso generalizado de las TIC.

En este sentido, en este documento se presenta una de las principales actividades realizadas, centrada en el desarrollo de un marco de apoyo psicoeducativo a estudiantes con diversidad funcional. En concreto, se ilustrará un desarrollo integrado en el curso "Descubriendo la plataforma”, realizado en la plataforma dotLRN (Santos et al., 2007), orientado a la familiarización del estudiante con el entorno virtual de aprendizaje y los diferentes servicios y recursos de apoyo asociados. El curso se ha implementado a través de la especificación educativa IMS Learning Design (IMS-LD) (IMS, 2003). Esta especificación ha sido elegida porque admite cualquier modelo psicoeducativo y permite describir diferentes flujos de aprendizaje manejando varios elementos (roles, actividades, entornos, recursos, propiedades, condiciones, servicios de monitorización y de notificación) (Koper \& Tattersall, 2005). IMS-LD soporta ocho tipos de adaptación que pueden realizarse en el sistema e-learning, como pueden ser (Burgos et al., 2007): I) basadas en la interfaz, II) caminos de aprendizaje alternativos, III) centradas en los contenidos, IV) soporte a la resolución interactiva de problemas, V) filtrado de información, VI) orientadas a grupos de usuarios y vii) evaluación, así como cambios inmediatos requeridos durante la interacción (Van Rosmalen y Boticario, 2005). Asimismo se ha utilizado GRAIL (Gradient-lab RTE for Adaptive IMS-LD in dotLRN) desarrollado por el Departamento de Ingeniería Telemática de la Universidad Carlos III de Madrid (Escobedo et al, 2007), para testear las unidades de aprendizaje desarrolladas a través de la especificación IMSLD. Se ha empleado GRAIL porque dotLRN es el sistema de gestión de aprendizaje donde se implementará el marco de apoyo diseñado en EU4ALL dentro del piloto de la UNED. GRAIL fue diseñado y desarrollado con el objetivo de ser utilizado dentro de una comunidad de dotLRN (p.e. usuarios compartiendo recursos, foros, calendario, etc). El curso desarrollado es una instancia de una de esas comunidades, y las unidades de aprendizaje que lo componen son uno de los recursos a los que se puede acceder a través de la comunidad de dotLRN (Fuente et al., 2007).

El trabajo presentado en este documento propone una nueva aproximación en la elaboración de diseños instruccionales adaptados a la diversidad funcional y cognitiva de los usuarios. No existen evidencias de que este apoyo se haya prestado 
E. Campo; M. Saneiro; M. Montecelo; E. Raffenne; A. Rodriguez; O. Santos; J. Boticario Apoyo AdAPtativo Basado EN IMS-LD y Estrategias Psico-EDUCATIVAS PARA LA FAMILIARIZACión...

con anterioridad, lo que supone un valor innovador de este proyecto, ya que en el curso desarrollado, los criterios de accesibilidad y usabilidad se han considerado tanto en la estructura del mismo como en el acceso a los contenidos, actividades y recursos en él englobados.

En las siguientes secciones de este artículo se describe la metodología empleada para la implementación del diseño instruccional realizado, así como la selección y elaboración de los diferentes contenidos y recursos de apoyo.

\section{DISEÑO DEL CURSO}

\section{Aproximación Psicoeducativa}

La aproximación psicoeducativa que subyace tanto al diseño instruccional de los contenidos educativos como a los propios recursos de apoyo englobados en el curso, se enmarca dentro del modelo cognitivo-constructivista en el que el aprendiz tiene un rol activo en el aprendizaje, y éste es considerado como un producto tanto de sus procesos cognitivos como de los factores propios del entorno en el que éste se produce. El objetivo final es que el alumno adquiera las estrategias de aprendizaje necesarias para realizar un proceso de aprendizaje autorregulado, autónomo destinado a mejorar sus competencias y a implementar aquellas que no posee o están funcionando de forma deficitaria como consecuencia de la discapacidad que presenta (p.e. dificultades en el procesamiento de la información, problemas de memoria, razonamiento, atención, lenguaje, etc.).

Desde esta perspectiva, el primer paso a la hora de abordar la creación de un curso adaptado a las necesidades específicas del usuario, fue la identificación de aquellas estrategias psicoeducativas que el sujeto debía utilizar adecuadamente en una situación de aprendizaje, de cara a optimizar sus resultados. Estas estrategias de aprendizaje han sido agrupadas en una taxonomía elaborada por algunos coautores de este artículo a partir de la revisión de taxonomías realizadas por diversos autores y perspectivas (Bloom, et al., 1984), (Ellis, 1993), (Wood, Woloshyn, y Willoughby , 1995), (Boulet, Savoie-Zajc, Chevrier , 1996), (Johnson, Graham y Harris, 1997), (Carmel y Markovitch, 1998), (Ridley, et al.,1992) y se agrupan fundamentalmente en torno a cuatro categorías:

- Estrategias cognitivas: utilizadas por los estudiantes para adquirir, mantener y recuperar conocimiento. Dentro de esta categoría se englobarán aquellas actividades destinadas a mejorar procesos cognitivos como seleccionar, organizar y almacenar información relevante de cara a la resolución de una tarea. 
E. Campo; M. Saneiro; M. Montecelo; E. Raffenne; A. Rodriguez; O. Santos; J. Boticario Apoyo AdAPTATIVo BASADO EN IMS-LD y ESTRATEGIAS PSICO-EDUCATIVAS PARA LA FAMILIARIZACión...

- Estrategias metacognitivas: empleadas por el sujeto para "aprender a aprender". Dentro de éstas se englobarían aquellas actuaciones destinadas a comprobar, planificar, revisar y evaluar la idoneidad del conocimiento adquirido por el aprendiz.

- Estrategias de interacción y comunicación: destinadas a regular la interacción del sujeto con otros de su entorno, facilitando el control que éste posee en situaciones en las que algunos aspectos psicológicos como son la ansiedad podrían interferir en sus habilidades de participación.

- Estrategias afectivas: que permitirían al individuo controlar las interferencias emocionales que podrían impactar en su proceso de aprendizaje, como son la necesidad de refuerzo constante, la aceptación de críticas en sentido positivo,...

Diversas investigaciones señalan la importancia de incluir estas estrategias en un programa de aprendizaje virtual (Barba, 1993 y Thornburg, 1991). Los aprendices que participaron en cursos en los que estas estrategias han sido consideradas no sólo obtuvieron mejores resultados, sino que además éstos se mantuvieron en el tiempo después de meses. De acuerdo con Merrill (1997) y Whitlock (2001) el aprendiz debe aprender a manejar estas estrategias y por tanto deben ser consideradas a lo largo de la metodología de instrucción, integrándose en las diferentes actividades académicas como son práctica, asesoramiento, comunicación, evaluación, etc. Además el aprendizaje en un entorno virtual requiere que el usuario maneje estrategias específicas como son habilidades para compartir conocimiento, para aprender a distancia, para reflexionar por sí mismo, controlar y monitorizar su proceso de aprendizaje. Precisamente estas son las habilidades que caracterizan las necesidades de un estudiante en el cada vez más imperante marco del aprendizaje permanente (Knapper y Cropley, 2000).

\section{Integración de estrategias en el diseño instruccional}

El apoyo psicoeducativo en el diseño del curso se ha centrado en la inclusión de las estrategias mencionadas a lo largo del flujo de aprendizaje, generándose recursos y contenidos que permitiesen el desarrollo y la mejora de estas estrategias esenciales de cara a la adquisición de conocimientos y participación con el entorno de aprendizaje y los diferentes agentes en él involucrados.

Las adaptaciones realizadas en el curso se basan en la configuración de elementos (actividades, contenidos, servicios) en un periodo específico, para incrementar 
el grado de rendimiento del alumno en un determinado dominio. Los contenidos del curso han sido diseñados considerando el criterio de reusabilidad por parte de diferentes usuarios que pueden compartir en un momento determinado las mismas necesidades psicoeducativas. De forma específica se han generado contenidos y recursos destinados a personas con déficit de atención, dislexia y déficits auditivos. Este colectivo comparte diversas dificultades tales como limitaciones en el procesamiento de la información, memoria, atención, razonamiento, elaboración y comprensión de textos, pobreza de vocabulario etc. (Lerner, 1997). Como consecuencia, estos sujetos pierden información relevante de cara a abordar las diferentes actividades académicas y sociales que se le plantean en el entorno de aprendizaje y en el mundo real.

Teniendo en cuenta esta información se elaboró el curso "Descubriendo la plataforma". Se trata de una versión evolucionada basada en una versión anterior del citado curso, desarrollada en otro de los proyectos del grupo aDeNu, el proyecto ALPE, en que se siguió una metodología de creación cursos accesibles y reusables desarrollados en SCORM (Santos et al, 2007). La versión actual ha sido implementada en IMS-LD y enriquecida a través de la mejora en la reusabilidad y adaptación de contenidos, así como la integración de estrategias psicoeducativas. El objetivo del curso es la familiarización del estudiante con el entorno virtual de aprendizaje, con flujos dinámicos adaptados al perfil del usuario que considera características personales, estrategias de aprendizaje y técnicas de adaptación. El perfil corresponde al de personas con dislexia, déficit auditivo y déficit de atención, con dificultades para recordar información relevante, fechas de entrega, problemas aislados de comprensión lectora, que deciden retomar sus estudios tras un largo periodo de abandono. Para determinar el perfil se desarrollaron cuestionarios sobre:

- Dificultades para realizar las diferentes actividades (p.ej. presentación en público, dificultades para la planificación, etc.).

- Cuestionario de Estilos de aprendizaje de Felder (Felder y Silverman, 1988).

- Conocimientos previos de utilización de recursos virtuales.

Tomando como base la información facilitada por el alumno en estos cuestionarios, si el usuario es considerado como susceptible de beneficiarse de un curso de esta naturaleza adaptado en flujo y contenidos a sus necesidades específicas, el sistema le ofrecerá la posibilidad de realizar el curso en su modalidad adaptada. Otros alumnos en los que no se detecte esta necesidad, o que no deseen realizar 
el curso en su modalidad adaptada, lo realizarán en una versión en la que no ha sido incorporada ningún tipo de adaptación. En la figura 1 se muestran los flujos alternativos de aprendizaje que el sistema le ofrece al alumno.

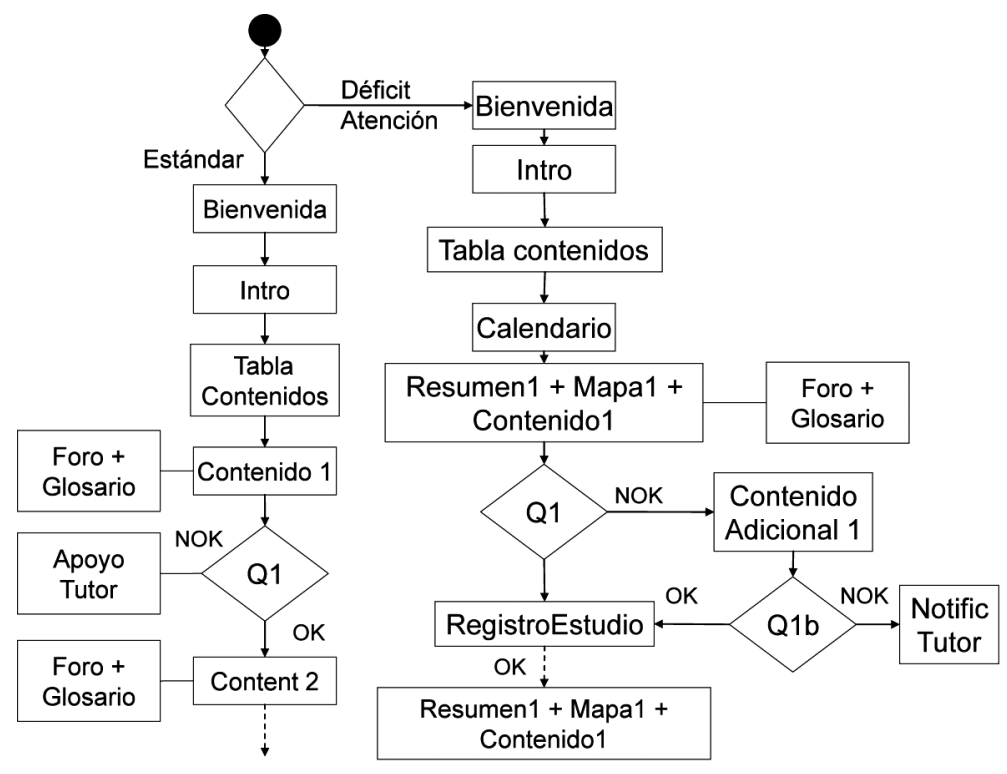

Figura 1. Diagrama de actividades

\section{- Interfaz del curso}

La interfaz del curso se ha creado a través del IMS-LD player para dotLRN y está dividido en tres secciones (ver figura 2):

Parte superior izquierda de la interfaz: en el que aparece el índice de los contenidos del curso.

Parte central derecha: un segundo framework en el que el estudiante podrá visualizar los contenidos de la sección que está consultando en ese momento.

Parte inferior izquierda de la interfaz: en el que aparecerán los recursos de apoyo asociados a cada unidad. 
E. Campo; M. Saneiro; M. Montecelo; E. Raffenne; A. Rodriguez; O. Santos; J. Boticario Apoyo AdAPtativo Basado EN IMS-LD y Estrategias Psico-EDUCATIVAS PARA LA FAMILIARIZACión...

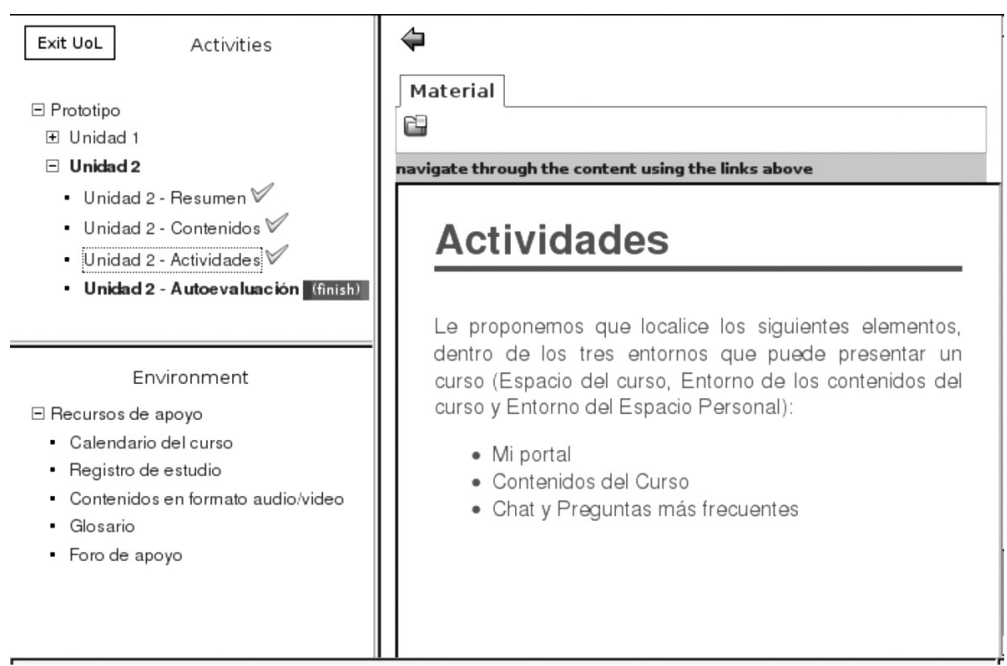

Figura 2. Interfaz del curso

\section{- $\quad$ Estructura del Curso}

El curso está compuesto de nueve unidades de aprendizaje en las que se han integrado diferentes adaptaciones, tanto en contenidos como en recursos de apoyo, para dar respuesta a las necesidades específicas del colectivo mencionado anteriormente. El usuario puede hacer uso de estos recursos para mejorar sus estrategias dependiendo de sus objetivos, competencias, contexto, velocidad y capacidad de aprendizaje.

La estructura del curso es la siguiente:

- Índice de materiales de aprendizaje

El sistema facilita un índice de las unidades de aprendizaje que el alumno deberá superar de manera secuencial como se muestra en la figura. 3 


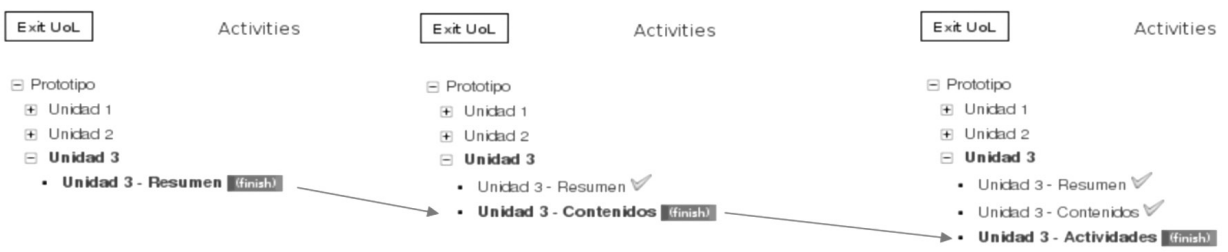

Figura 3. Secuencia de las unidades de aprendizaje

El diseño de las unidades de aprendizaje, permitirá al alumno, como se muestra en la Figura. 4, tener una idea de conjunto sobre la organización y estructuración de las diferentes tareas que tendrá que abordar, estudio de contenidos teóricos, aspectos relevantes de la temática recogidos en los diferentes capítulos de cada tema, actividades de carácter práctico, sistema de evaluación y recursos de apoyo asociados.

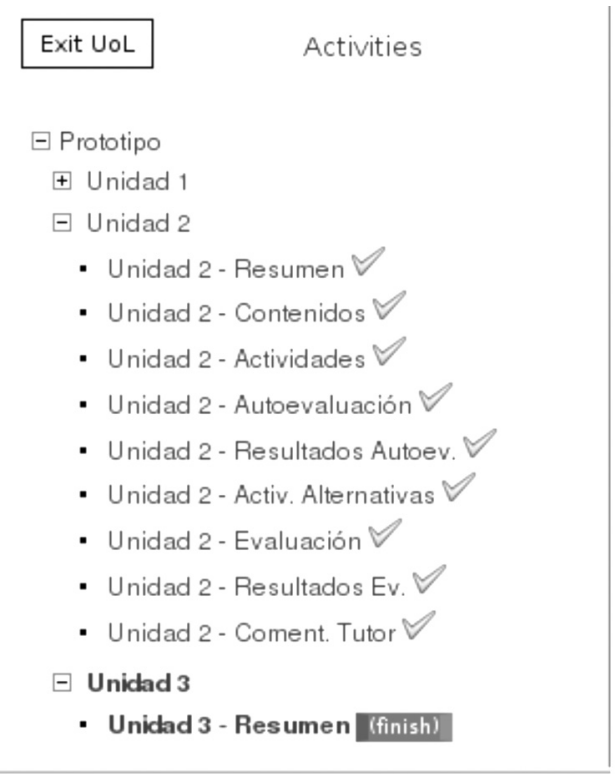

Figura 4. Estructura de la unidad de aprendizaje

- Resumen y mapa conceptual

Tanto el resumen como los mapas conceptuales aparecen al comienzo de cada unidad y están orientados a facilitar al estudiante información resumida sobre cuales 
E. Campo; M. Saneiro; M. Montecelo; E. Raffenne; A. Rodriguez; O. Santos; J. Boticario Apoyo AdAPtativo Basado en IMS-LD y Estrategias Psico-edUCATIVAS PARA LA FAMILIARIZACión...

con los objetivos específicos de cada unidad, así como los principales conceptos que en ella se tratarán y la interrelación que existe entre ellos.

\section{Unidad 4: Cómo usar el calendario}

\section{Resumen}

En este tema nos centraremos en el segundo elemento del espacio personal, el calendario. Este recurso calendario le permite planificar, organizar temporalmente y controlar su actividad académica y relaciones personales con sus compañeros o profesores.

\section{Mapa conceptual}

Unidad 4: El espacio personal: El calendario

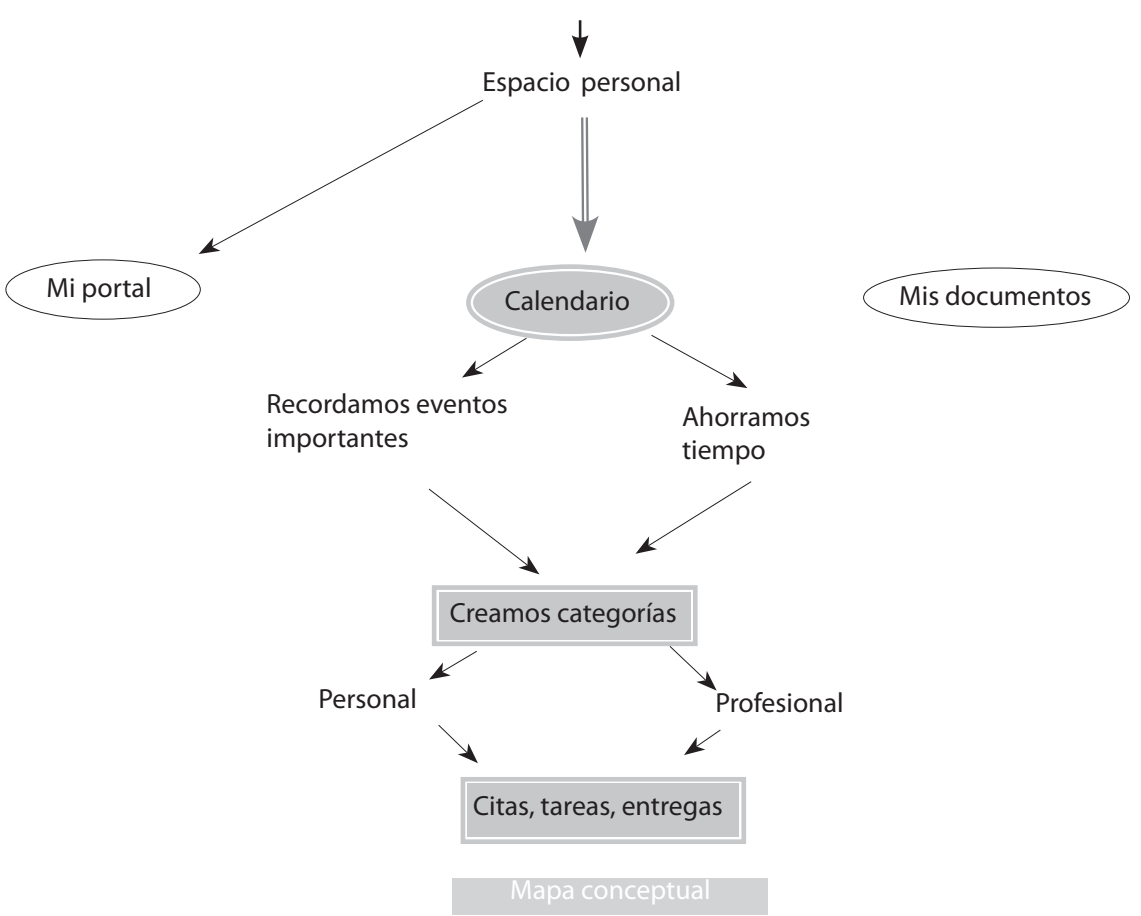

Figura 5. Resumen y mapa conceptual 


\section{- Contenido Teórico}

Como se ha mencionado anteriormente, el contenido teórico está dividido en nueve Unidades de Aprendizaje en las que se han realizado adaptaciones destinadas a facilitar el desempeño de personas con dislexia, déficit de atención y déficit auditivo. Específicamente las adaptaciones de formato realizadas son:

- contenidos redactados en formato de fácil lectura: centrados en contenidos relevantes, evitando jergas, abreviaturas, lenguaje demasiado complejo,

- aumento de tamaño de la fuente,

- ampliación de espaciado entre párrafos para no saturar a nivel cognitivo ya que este colectivo presenta déficit en el procesamiento de la información,

- utilización de lecciones cortas dirigidas a los aspectos relevantes y esenciales del contenido,

- utilización de colores asociados a cada aspecto teórico a tratar, para facilitar la localización y asimilación de los mismos,

- material enriquecido con iconos,

- disponibilidad de material didáctico en formato adaptado (vídeo y audio).

En la figura 6 se muestra un ejemplo de contenidos didácticos, redactados en lectura fácil, utilizando colores y enriquecidos con iconos. 
E. Campo; M. Saneiro; M. Montecelo; E. Raffenne; A. Rodriguez; O. Santos; J. Boticario APOyo AdAPTATIVo BASAdo EN IMS-LD y ESTRATEGias PSICO-EDUCATIVAS PARA LA FAMILIARIZACióN...

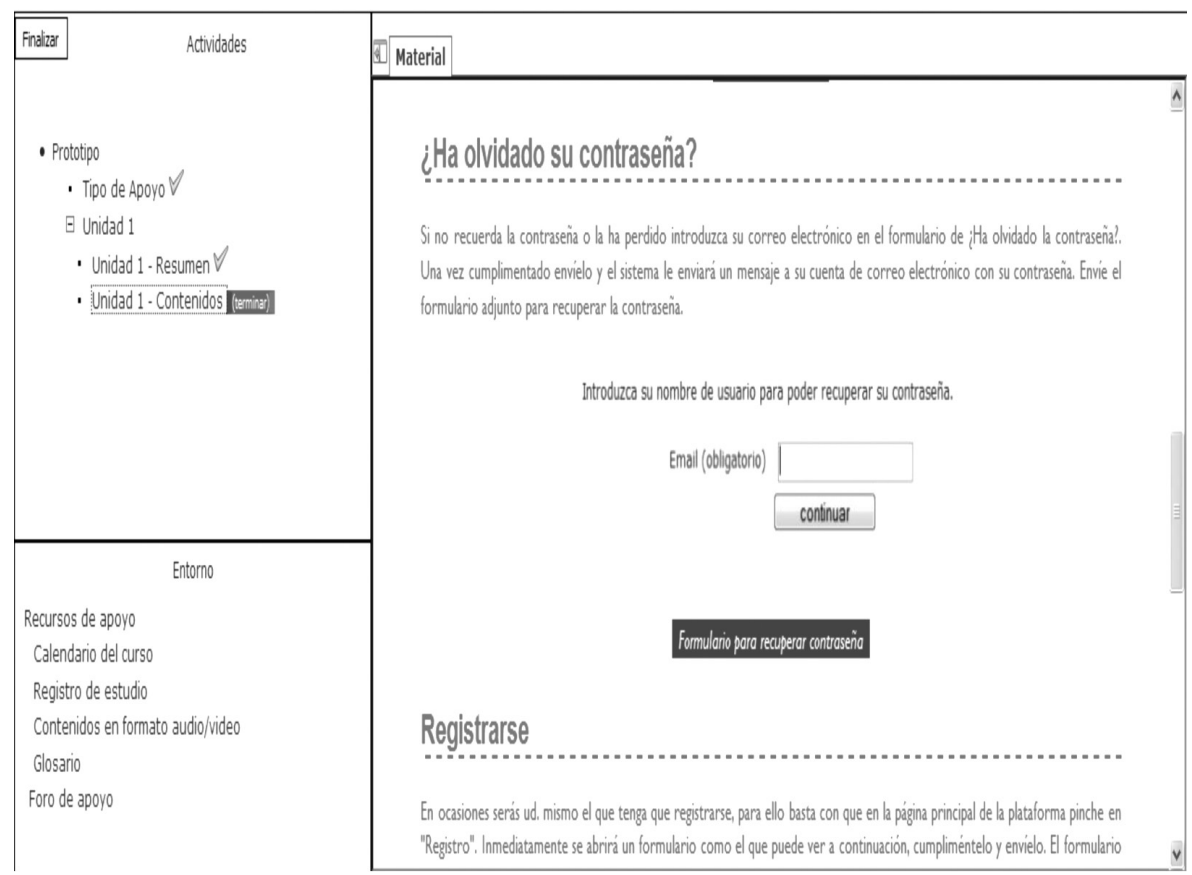

Figura 6. Contenidos adaptados

- Actividades de entrenamiento estándar y alternativas

Estas actividades se proponen al aprendiz después de cada unidad y están diseñadas para ayudarle a asimilar los contenidos poniendo en prácticas los conocimientos adquiridos. Existen dos tipos de actividades, el primer tipo es el de actividades estándar que se muestra en la Figura 7. Estas actividades serán realizadas por todos los alumnos. 


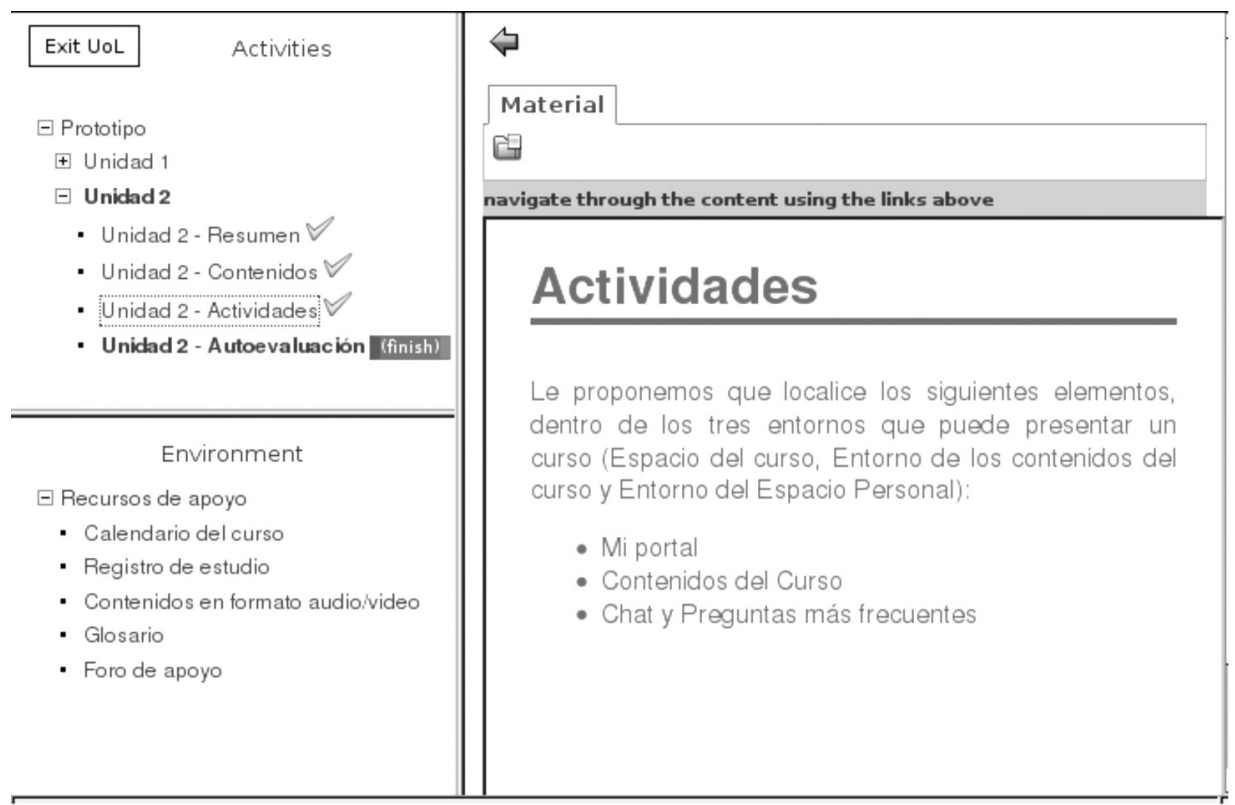

Figura 7. Actividades estándar

- Cuestionarios de evaluación

El sistema, una vez finalizada la revisión de los contenidos teóricos y la puesta en práctica de los mismos a través de la realización de las actividades de carácter práctico propuestas, ofrecerá al alumno la posibilidad de evaluar sus conocimientos a través de un cuestionario de evaluación, que le permitirá monitorizar si la adquisición de conocimientos se está produciendo de forma adecuada. Teniendo en cuenta que el rendimiento del alumno en esta prueba puede verse afectado por el propio diseño de la prueba de evaluación, debido a que por ejemplo es demasiado larga, requiere demasiado esfuerzo para su realización, etc., se han incluido diferentes tipos de cuestionarios adaptados a las necesidades del alumno. Las adaptaciones se refieren tanto a la extensión del mismo como a su formato, facilitándose diferentes formas de respuesta, verdadero/falso, elección múltiple, correspondencia, respuestas cortas, etc. Esta adaptación permitirá al alumno rendir de acuerdo con sus capacidades seleccionando la opción que mejor se adapte a su perfil y necesidades. En la figura 8 se muestra una actividad de evaluación en la que el alumno debe decidir si la idea principal que se le presenta relacionada con el contenido de la unidad de aprendizaje es verdadero o falso. 


\section{Autoevaluación}

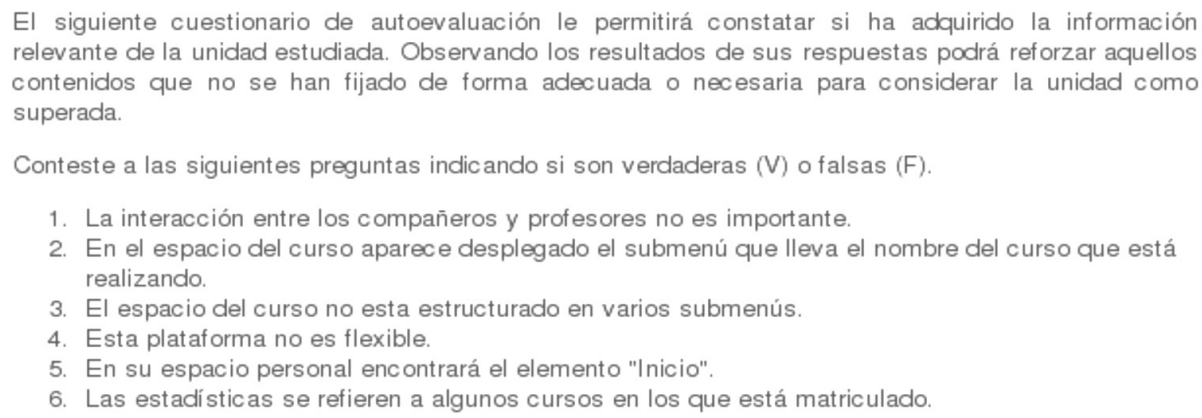

Respuestas:

1. $\mathrm{F}-\boldsymbol{\nabla}$ ok

2. $\mathrm{F}[\boldsymbol{\nabla}$ ok

3. $\mathrm{F}-\mathrm{ok}$

4. $\mathrm{F} / \boldsymbol{\nabla}$ ok

5. $\mathrm{F}[\boldsymbol{\nabla}$ ok

6. $\mathrm{F}[\boldsymbol{\nabla}$ ok

Figura 8. Cuestionario de autoevaluación

- $\quad$ Actividades alternativas

Si el alumno no consigue superar las actividades estándar propuestas en el curso, el sistema le ofrecerá la posibilidad de revisar contenidos adicionales y volver a poner en práctica sus conocimientos a través de la realización de otras actividades alternativas, en las que se han diseñado tareas que permitan al sujeto poner en práctica sus estrategias de aprendizaje (p.e. buscar ideas relevantes, contactar con el foro para aclaración de dudas, revisión de contenidos adicionales, planificar una actividad en el calendario, etc.). En la figura 9 se muestra una actividad alternativa que se ofrecerá al alumno para que aprenda a seleccionar y codificar las principales ideas de un tema y las estructure en un esquema o mapa conceptual que le permita relacionar los diferentes conceptos de esa unidad específica. 


\section{Actividades Alternativas}

Asocie el número que corresponda a cada respuesta, para que represente el mapa conceptual de la unidad.

Nota: Cuando haya completado el esquema, puede ver las respuestas que se encuentran en el fondo de la página

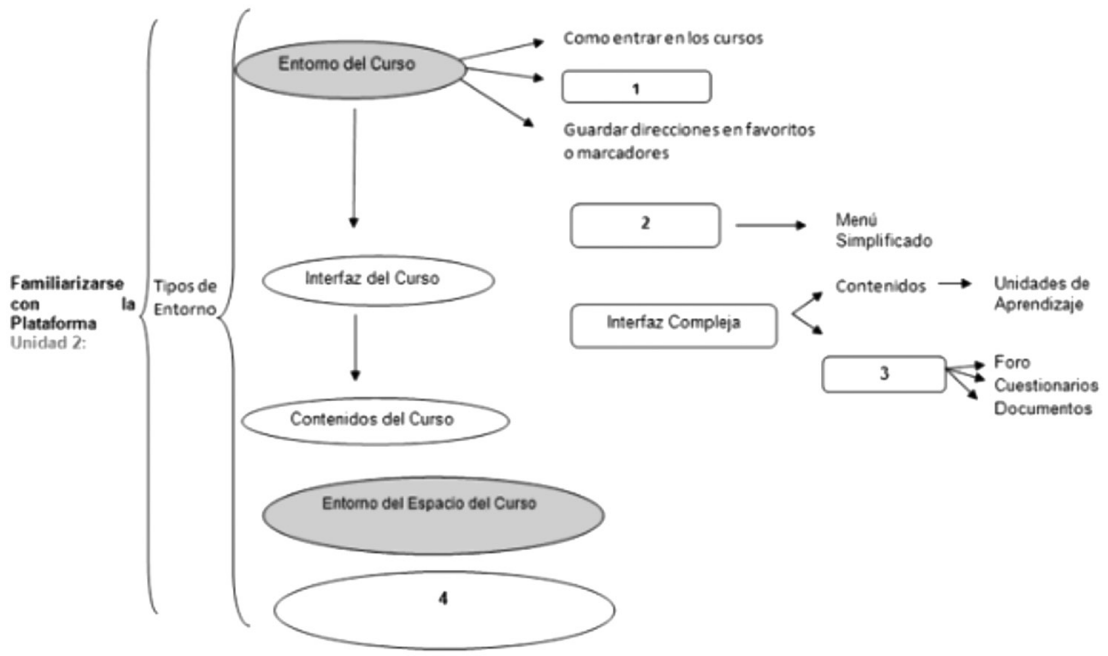

Figura 9. Actividades alternativas

- Cuestionario final de evaluación

Una vez que el alumno ha realizado la actividad alternativa que le propuso el sistema, se le propone cumplimentar un cuestionario de evaluación final. Del mismo modo que en el caso de los cuestionarios de evaluación intermedios, el formato del cuestionario de evaluación final ofrecerá diferentes posibilidades de respuesta, cumplimentar un cuadro, correspondencia, elección múltiple, etc. Si el alumno lo supera habrá finalizado la unidad de aprendizaje y podrá avanzar a la siguiente. Tras la cumplimentación, tanto del cuestionario intermedio como del cuestionario final de evaluación, el sistema ofrecerá feedback sobre sus resultados, donde se ha producido el error, etc. Esto permitirá al alumno revisar sus conocimientos y mejorar o profundizar aquellos que todavía están incompletos o son incorrectos. El feedback no sólo permite mejorar el conocimiento, también ejerce un efecto positivo en el 
nivel de autoconfianza y autoestima del individuo porque le permite constatar que su aprendizaje está siendo correctamente autorregulado por el mismo, aumentando por tanto su confianza en sus capacidades y estrategias de estudio. En la figura 10 se muestra un ejemplo de mensaje positivo al alumno tras haber superado el cuestionario de evaluación final de una unidad de aprendizaje.

\section{Resultados de la autoevaluación}

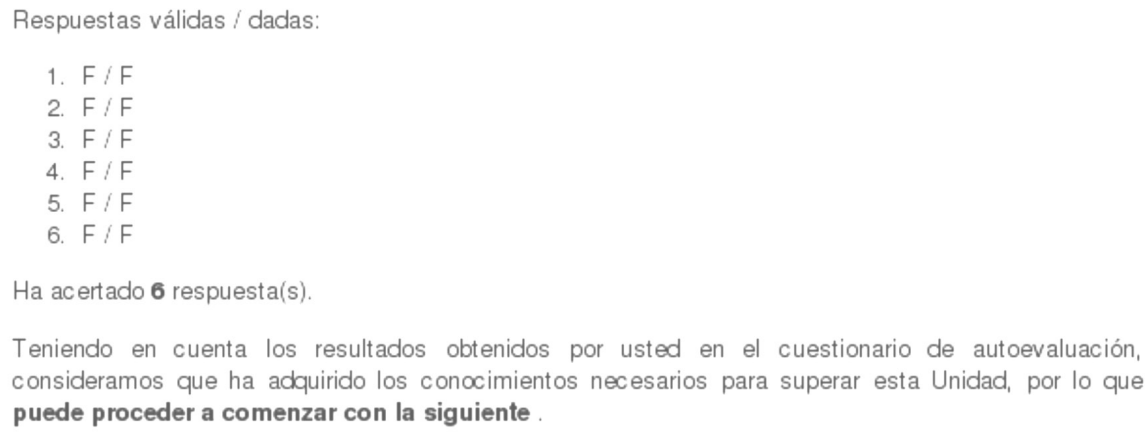

Figura 10. Feedback de evaluación

- Comentarios del tutor/profesor

El curso está diseñado de forma que, además de incorporar un funcionamiento que se adapta automáticamente al perfil y el desempeño del estudiante, se apoye y fomente la comunicación entre el profesor y el alumno. Este es el caso de la situación en la que el alumno, tras serle ofrecidos contenidos o actividades alternativas, no supere el cuestionario de evaluación final, el tutor le enviará un mensaje con las orientaciones adecuadas para mejorar sus resultados, o incluso recomendarle contactar con un profesional especializado en la problemática que el alumno presenta. Así, a través de este recurso (ver figura 11) el profesor podrá enviar feedback al alumno, monitorizando en todo momento su aprendizaje, reconduciéndole si es necesario a la realización de nuevas actividades, revisión de nuevos contenidos, etc. 


\section{Comentarios del tutor sobre esta unidad}

Si existen, los comentarios del tutor a respecto de su progreso y resultados con esta unidad aparecerán a continuación:

Comentario de prueba personalizado para el alumno U. 5

Figura 11. Comentarios del tutor

- Recursos de apoyo globales

Además de los recursos integrados a lo largo de las diferentes actividades que el curso contiene, se han diseñado recursos de apoyo globales destinados a mejorar otras estrategias necesarias para el alumno de cara a un desenvolvimiento óptimo a nivel académico. Los principales recursos son los siguientes:

$\checkmark$ Calendario de trabajo: A través del recurso, que se muestra en la figura 12, el estudiante podrá chequear todas las actividades programadas en el curso, el tiempo destinado a cada una de ellas, cuestionarios de evaluación, fechas límites de entrega de tareas asignadas, etc. Este recurso permitirá al alumno ejercitar sus estrategias de organización y planificación del tiempo destinado para cada actividad del curso, evitando confusiones, olvidos, etc. 


\section{Calendario de estudio}

\begin{tabular}{|c|c|c|c|c|c|}
\hline Lunes 1 & Martes 2 & Miércoles 3 & Jueves 4 & Viernes 5 & Sábacdo 6 \\
\hline \multicolumn{6}{|l|}{$\begin{array}{l}\text { Estudio T1, Hacer } \\
\mathrm{C} 1 \text { y Entrega T1. } \\
\text { Estudio T2, Hacer } \\
\mathrm{C} 2 \text { y Entrega T2. }\end{array}$} \\
\hline & $\begin{array}{l}\text { Estudio T3, Hacer } \\
\text { C3y Entrega T3. } \\
\text { Estudio T4, Hacer } \\
\text { C4y Entrega T4. }\end{array}$ & & & & \\
\hline & & $\begin{array}{l}\text { Estudio T5, Hacer } \\
\text { C5 y Entrega T5. } \\
\text { Estudio T6, Hacer } \\
\text { C6 y Entrega T6. }\end{array}$ & & & \\
\hline & & & $\begin{array}{l}\text { Estudio T7, Hacer } \\
\text { C7 y Entrega T7. } \\
\text { Estudio T8, Hacer } \\
\text { C8y Entrega T8. }\end{array}$ & & \\
\hline & & & & $\begin{array}{l}\text { Estudio T9, Hacer } \\
\text { Cgy Entrega T9. }\end{array}$ & \\
\hline & & & & & $\begin{array}{l}\text { EVALUACIÓN } \\
\text { FINAL. }\end{array}$ \\
\hline
\end{tabular}

Figura 12. Calendario de estudio

- Registro de estudio: Tras consultar el contenido teórico de cada unidad, este recurso le permitirá al estudiante reflejar cuáles son sus dudas a consultar, monitorizar el tiempo destinado a ello, problemas encontrados en el estudio, etc. El registro de estudio permitirá al alumno poner en práctica estrategias como monitorización, manejo del tiempo, codificación de errores, atribución causal de los mismos comprobando si el tiempo destinado a estudio ha sido el suficiente, estrategias de clarificación de dudas que presenta, cómo resolverlas, etc. 
Registro de estudio

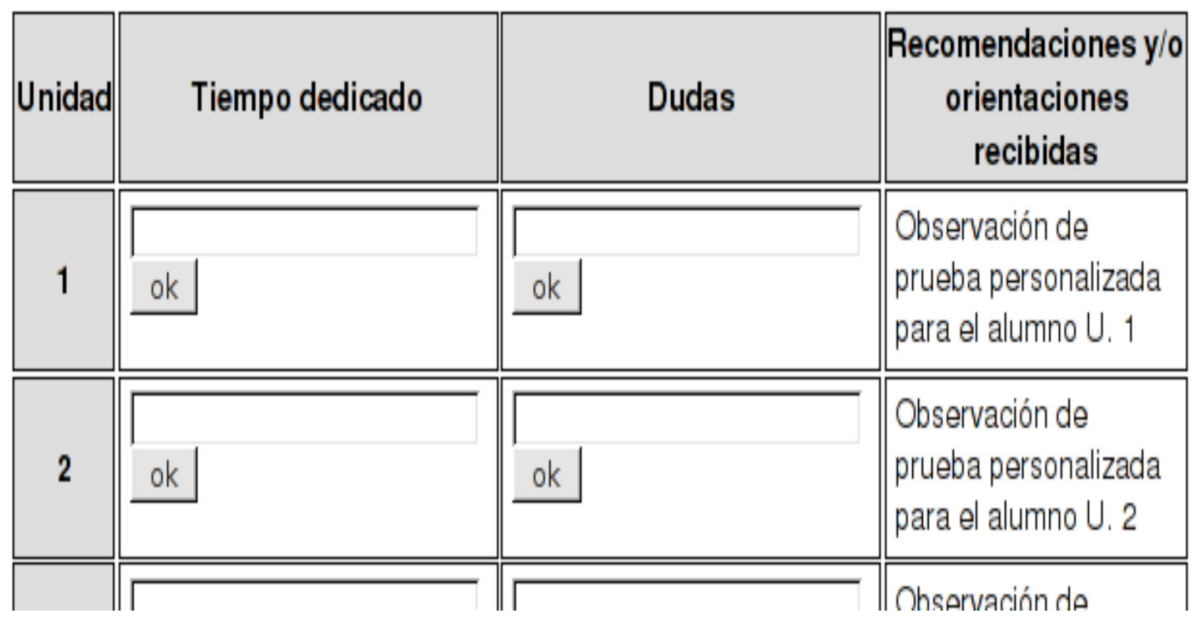

Figura 13. Registro de estudio

- Glosario: El glosario, elaborado para el curso, ayudará al alumno a conocer cuáles son las palabras técnicas del tema a tratar. Esto no sólo aumenta el vocabulario del alumno (que recordemos es deficitario en el colectivo seleccionado), sino que además le permitirá evitar confusiones, codificando y elaborando información de forma adecuada a la hora de elaborar contenidos o compartirlos con otros. 
E. Campo; M. Saneiro; M. Montecelo; E. Raffenne; A. Rodriguez; O. Santos; J. Boticario

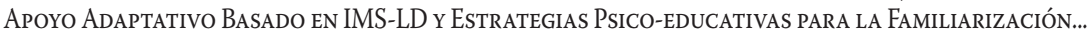

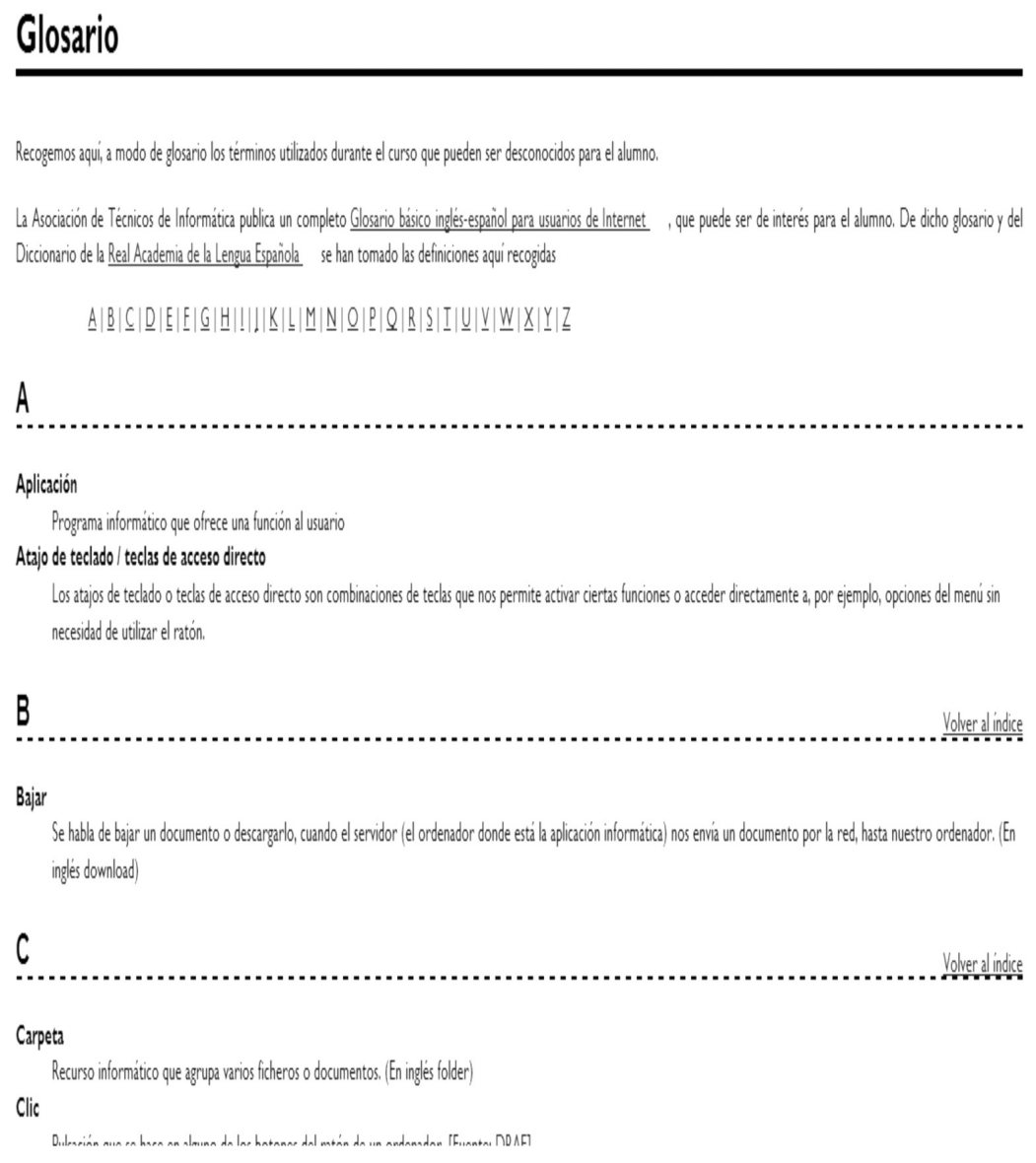

Figura 14. Glosario

- Foro y Chat: El foro es un recurso importante de cara a que el alumno ponga en práctica sus estrategias de comunicación e interacción. Este recurso, no es específico del curso sino que está disponible en la plataforma dotLRN para todos los usuarios, independientemente de si presentan o no discapacidad. Junto con el Chat permiten mantener comunicación síncrona y asíncrona con todos los participantes en el curso. Constituyen una importante herramienta de trabajo colaborativo a través de la cual el alumno aprenderá a compartir puntos de vista con otros, aceptar críticas, resolver dudas y en general interaccionar mediante la utilización de un lenguaje adecuado, desarrollando así sus estrategias afectivas y de comunicación e interacción. 
E. Campo; M. Saneiro; M. Montecelo; E. Raffenne; A. Rodriguez; O. Santos; J. Boticario Apoyo AdAPTATIVo BASADO EN IMS-LD y ESTRATEGIAS PSICO-EDUCATIVAS PARA LA FAMILIARIZACión...

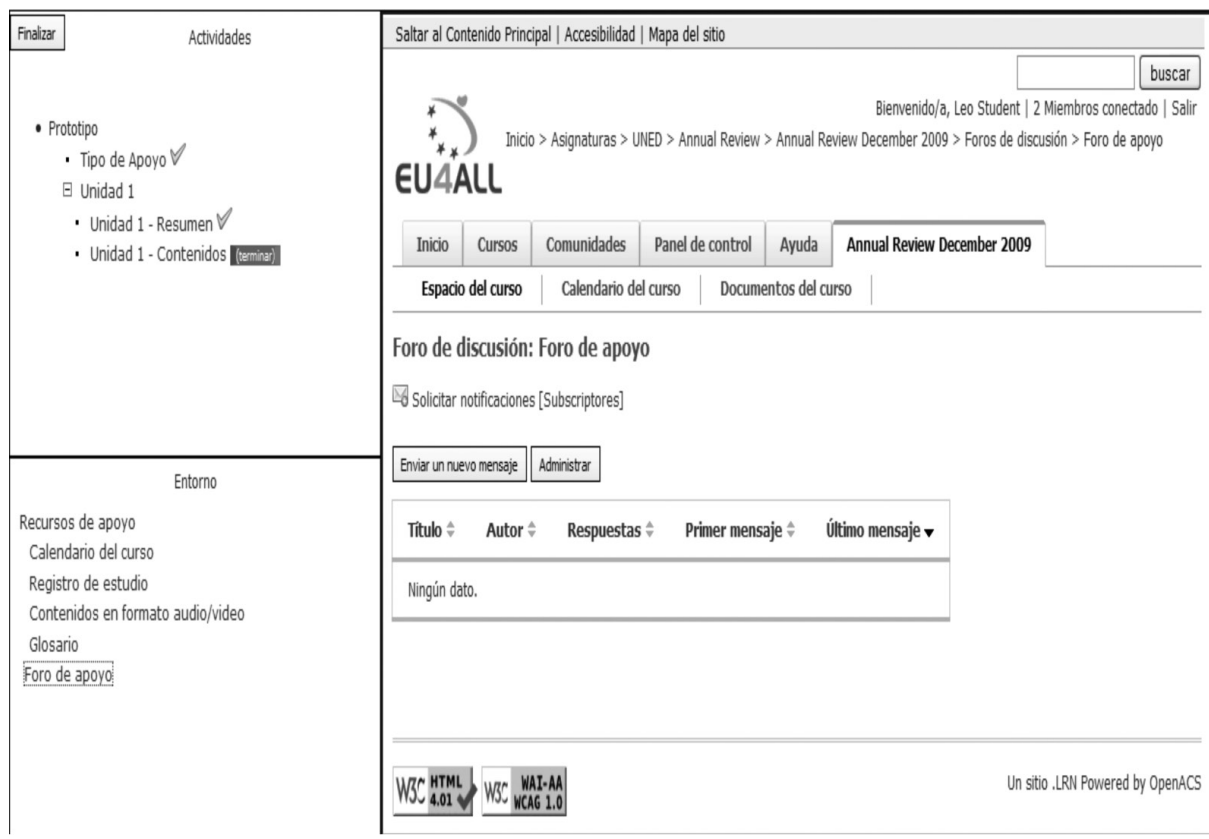

Figura 15. Foro de comunicación

\section{- Calendario}

Este recurso, al igual que en el caso anterior, es un recurso general disponible en dotLRN para todos los alumnos matriculados en el curso. Permite la creación de citas o eventos relacionados con la programación del curso y supone un espacio de entrenamiento para la puesta en práctica de las estrategias de planificación y organización, esenciales para alcanzar el éxito en el curso, en el cual el alumno puede crear categorías de eventos como son: entrega de trabajos, tutorías, sesiones de puesta en común de ideas a través del chat o foro, etc. En la figura 16 se muestran sus funcionalidades. 


\section{Crear categorías de citas}

\section{El calendario nos permite crear categorías para distintos tipos de citas. \\ Podremos crear la categoría "personal" para citas o eventos particulares. \\ Para ello debemos acceder a la opción "Administrar categoría".}

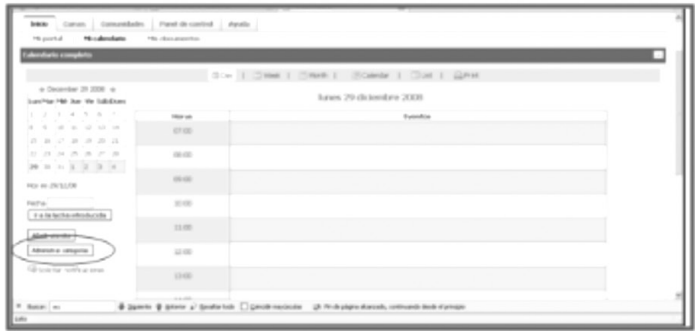

Añadir categoría al calendario

Podemos crear cuantas categorías nec esitemos.

Se pueden denominar como queramos: si son personales, laborales, etc

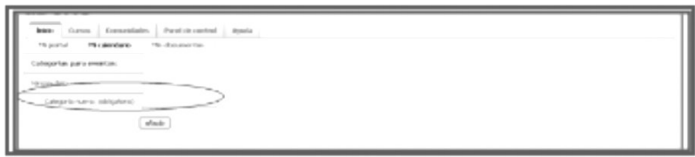

Categoria del calendario

Figura 16. Calendario

- Contenido alternativo en vídeo y audio

Teniendo en cuenta las necesidades específicas de los usuarios que serán destinatarios del curso, entre los que se encuentran personas con déficit auditivo, dislexia y déficit de atención, se ha desarrollado una versión adaptada de los contenidos teóricos de cada asignatura, tanto en formato video con subtítulos como en formato audio. La disponibilidad de afrontar los contenidos en un formato adaptado a las necesidades especiales de estos sujetos mejora el proceso de codificación y elaboración de la información, no sólo porque pueden acceder al mismo en su totalidad, sino porque además pueden seleccionar uno u otro dependiendo de su estilo de aprendizaje más visual o más auditivo. 
E. Campo; M. Saneiro; M. Montecelo; E. Raffenne; A. Rodriguez; O. Santos; J. Boticario Apoyo AdAPTATIVo BASAdo EN IMS-LD y Estrategias PSICO-EDUCATIVAS PARA LA FAMILIARIZACión...

\begin{tabular}{|c|c|c|c|c|c|c|}
\hline $\begin{array}{l}\text { Estrategias de } \\
\text { aprendizaje }\end{array}$ & Chat & Foro & Calendario & FAQ & $\begin{array}{l}\text { Contenidos } \\
\text { teóricos }\end{array}$ & $\begin{array}{l}\text { Actividades } \\
\text { de evaluación }\end{array}$ \\
\hline \multicolumn{7}{|l|}{ Cognitivas } \\
\hline Ensayo & $x$ & $x$ & & & $x$ & $x$ \\
\hline Organización & & & $x$ & & $x$ & \\
\hline Codificación & $x$ & $x$ & & $x$ & $x$ & \\
\hline Elaboración & & & & & $x$ & \\
\hline Síntesis & & & & $x$ & $x$ & $x$ \\
\hline Generalización & $x$ & & $x$ & & $x$ & $x$ \\
\hline \multicolumn{7}{|l|}{ Metacognitivas } \\
\hline $\begin{array}{l}\text { Focalización de } \\
\text { atención }\end{array}$ & $x$ & $x$ & $x$ & $x$ & $x$ & $x$ \\
\hline Planificación & & & $x$ & & $x$ & \\
\hline $\begin{array}{l}\text { Monitorización y } \\
\text { regulación }\end{array}$ & $x$ & $x$ & $x$ & $x$ & $x$ & $x$ \\
\hline Evaluación & & & & & & $x$ \\
\hline \multicolumn{7}{|l|}{ Interacción } \\
\hline Cooperación & $x$ & $x$ & & & & \\
\hline Clarificación & $x$ & $x$ & $x$ & & & $x$ \\
\hline \multicolumn{7}{|l|}{ Comunicación } \\
\hline Conversacional & $x$ & $x$ & & & & \\
\hline \multicolumn{7}{|l|}{ Afectivas } \\
\hline Autoestima & & & & & $x$ & $x$ \\
\hline $\begin{array}{l}\text { Cambio } \\
\text { Atribucional }\end{array}$ & $x$ & $x$ & & & & \\
\hline Autoeficacia & & & $x$ & & $x$ & $x$ \\
\hline Autocontrol & & & $x$ & $x$ & & $x$ \\
\hline
\end{tabular}

Tabla1. Estrategias y recursos de apoyo 


\section{INTEGRACIÓN TÉCNICA DEL CURSO EN LA ARQUITECTURA DE EU4ALL}

\section{IMS-LD y dotLRN}

Los proyectos EU4ALL y A2UN@ facilitan apoyo accesible y adaptativo para optimizar los esfuerzos realizados por el estudiante a lo largo del proceso de aprendizaje, orientados a la adquisición y aplicación de conocimientos, así como a la realización de las diferentes tareas académicas que debe abordar en el ámbito de la Educación Superior. El modelo de apoyo tiene como objetivo permitir un mejor uso de los contenidos de aprendizaje, recursos y servicios integrados en el curso diseñado, considerando para ello las necesidades y preferencias del usuario. Un mismo curso como hemos indicado anteriormente puede ser presentado de forma diferente a un grupo diverso de usuarios dependiendo de sus capacidades y necesidades específicas. Tomando como base éstas el sistema facilitará un espacio para la adquisición de conocimientos así como los recursos adaptados necesarios para alcanzar un rendimiento óptimo de esos usuarios.

Siguiendo a Blöchl, Rumetshofer, y Wöß, (2003) adaptar un curso a las necesidades específicas del usuario implica que el sistema de aprendizaje debe: I) considerar los patrones de aprendizaje de cada estudiante II) crear un espacio de entrenamiento para aplicar el conocimiento adquirido, III) permitir una organización instruccional del material y IV) ofrecer la posibilidad de seleccionar dinámicamente la mejor opción para el estudiante, así como una secuencia de aprendizaje de acuerdo con las necesidades del estudiante en las diferentes etapas del curso. La adopción de esta aproximación tiene implicaciones técnicas importantes en el marco del proyecto EU4ALL, que se describirán en las siguientes secciones del documento.

\section{Servicio de Modelado de Usuario}

El principal apoyo tecnológico para adaptar flujo y contenidos de aprendizaje a las necesidades de los usuarios, es el manejo de las propiedades de los estudiantes a través del Modelo de Usuario (UM). Como comentamos anteriormente, la adaptación es esencial en cualquier entorno de aprendizaje virtual ya que el aprendizaje es por naturaleza, un proceso evolutivo que depende en gran medida de las características del aprendiz y de su evolución a lo largo del tiempo. De forma específica los usuarios del aprendizaje virtual presentan una gran variedad de intereses, nivel de experiencia en la utilización de recursos virtuales, etc. Tomando como base estas características los usuarios demandan un entorno adaptado a sus necesidades y preferencias 
E. Campo; M. Saneiro; M. Montecelo; E. Raffenne; A. Rodriguez; O. Santos; J. Boticario Apoyo AdAPTATIVo BASADO EN IMS-LD y ESTRATEGIAS PSICO-EDUCATIVAS PARA LA FAMILIARIZACión...

específicas. La idea principal desde la perspectiva de los proyectos EU4ALLy A2UN@ es que esas adaptaciones deben realizarse siguiendo unos estándares y deben estar centradas en las necesidades de los usuarios, que en ocasiones serán manejadas en términos de modelos de usuarios.

Para proporcionar apoyo psicoeducativo en un entorno inclusivo de aprendizaje, las preferencias de accesibilidad deben necesariamente ser consideradas. Esto implica que deben analizarse las necesidades y preferencias que presenta el usuario a la hora de interactuar tanto con los contenidos como con los dispositivos. De acuerdo con este planteamiento los usuarios deben ser modelados de acuerdo con sus preferencias a la hora de acceder a los recursos tecnológicos y electrónicos.

Cuando el estudiante accede a la plataforma, el sistema le presenta una serie de cuestionarios que debe cumplimentar. Los datos obtenidos a través de estas herramientas serán almacenados como propiedades en el perfil del usuario. Esta información será utilizada para facilitar contenidos adaptados de acuerdo con esos valores. El Modelo de Usuario (UM) es el repositorio central de EU4ALL de almacenaje y gestión de la información relacionada con el usuario. El apoyo dinámico facilitado a lo largo del proceso de aprendizaje vendrá determinado por el perfil del usuario disponible en dicho UM.

Las características del usuario se relacionan con los recursos que serán definidos en términos de condiciones de aprendizaje en el curso. Así las principales variables que han sido utilizadas en este curso para determinar el perfil de usuario son las siguientes:

- Datos demográficos: información personal (nombre, fecha y lugar de nacimiento...).

- Preferencias psicoeducativas: referidas a dificultades que encuentran los estudiantes a la hora de afrontar los diferentes contenidos y tareas diseñadas en los cursos (p.e. problemas de producción y comprensión de textos, déficit de atención, bajas habilidades lingüísticas, manejo de tiempo, etc.).

- Preferencias: generales (p.e. idioma) y preferencias de accesibilidad (p.e. vídeo subtitulado).

- Estilos de aprendizaje: establecidos en base a las dimensiones consideradas por Felder (percepción, procesamiento, comprensión, entrada sensorial) y recogidas a través del test “Inventario de Estilos de Aprendizaje” (Felder,1988). 
- Competencias: información referida a las herramientas, habilidades y competencias que el alumno presenta pata enfrentarse tanto a los contenidos teóricos (experiencia, objetivos,...) como a actividades relacionadas con la interacción con otros (p.e nivel de colaboración, comunicación, participación, iniciativa, etc.).

- Progreso: información referida al progreso del alumno en el curso (nivel de conocimiento adquirido, objetivos, intereses para cada objetivo de aprendizaje, etc.).

La información recogida a través del análisis de estas variables, permitirá al sistema ofrecer al alumno una ruta de aprendizaje adaptada a sus necesidades específicas.

Para facilitar la gestión de las preferencias psicoeducativas que el alumno presenta (p.e. lectura fácil, dificultades de organización, manejo del tiempo, producción y comprensión de textos, etc.), esta información ha sido codificada tomando como referencia una serie de parámetros creados a través del UM. Estos métodos permiten al Sistema de Gestión del Aprendizaje o Learning Management System (LMS), (o cualquier otra aplicación que utilice los servicios del UM) crear, leer y actualizar objetivos, intereses, competencias y preferencias de los usuarios independientemente de la estructura XML subyacente. Además estos métodos abstractos facilitan una "historia" de apoyo, ya que cuando una propiedad se actualiza sus datos históricos también, de forma que cualquier valor antiguo se mantiene para referencias futuras o estudios evolutivos.

Los valores relativos a estas variables se obtendrán a través del sistema que gestionará el aprendizaje (LMS) y serán compartidos por otros módulos a través del UM, como se indica en la figura 17. La información se almacena en formato XML, siguiendo los estándares. ISO/IEC 24751-2 "Access for all" personal needs and preferences for digital delivery" para las opciones de accesibilidad relacionadas con los contenidos electrónicos e IMS-LIP (Learner Information Package), para preferencias e información del estudiante. Una API basada en servicios web (WS) mediante el Simple Object Access Protocol (SOAP) facilita el acceso a los datos a otros sistemas satélites, como pueden ser el módulo de personalización de contenidos (CP), el sistema recomendador (RS) o el portfolio (ePMS). 
IMS-LD

$\operatorname{dotLRN}$

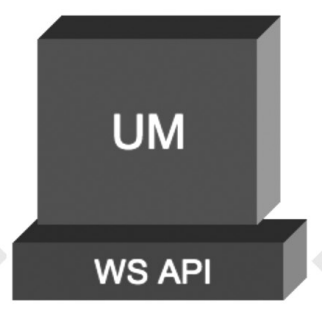

SOAP

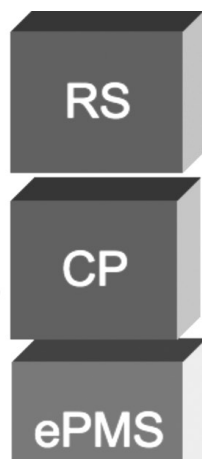

Figura 17. Arquitectura de EU4ALL desde la perspectiva del UM

\section{Integración del Modelo de Usuario con el Player IMS-LD}

El modelo de información de IMS-LD define el elemento "Propiedad Global Personal" como una propiedad perteneciente a una persona que puede presentar diferentes valores dependiendo de cada usuario, independientemente de la forma en la que se avance a lo largo de las diferentes unidades de aprendizaje. Las Propiedades Globales Personales son identificadas a través de la utilización de un identificador único (URI). En el marco de EU4ALL y A2UN@, las propiedades personales son almacenadas en el UM (preferencias, competencias, objetivos, intereses) y su localización puede expresarse a través de un Localizador Uniforme de Recursos (URL) que en ocasiones puede utilizarse como un URI.

Para acceder y utilizar una propiedad almacenada en el UM, el creador del diseño de aprendizaje identifica la propiedad utilizando el URI del UM. En tiempo de ejecución, el player IMS-LD resolverá el URI de la propiedad global y devolverá su valor desde el correspondiente proveedor, en este caso el servicio de Modelado de Usuario de EU4ALL. Del mismo modo si el valor de una propiedad cambia durante la ejecución del diseño de aprendizaje, el nuevo valor se volverá a enviar al servicio de Modelado de Usuario si la propiedad URI apunta hacia él.

\section{Servicio de personalización de contenidos}

Desde el punto de vista de la accesibilidad, los contenidos son personalizados en función de las preferencias y necesidades de accesibilidad informadas por el usuario a través del servicio de Personalización de Contenidos (CP). La personalización de 
contenidos es una de las principales características del marco de apoyo diseñado en EU4ALL, los contenidos serán personalizados de acuerdo a las preferencias y necesidades de los usuarios (p.e. texto alternativo) y el contexto (p.e. dependiendo del dispositivo que el usuario utilice para acceder a os contenidos y servicios).

La personalización de contenidos (CP) ha sido integrada en el player IMS-LD en el curso desarrollado en dotLRN. Cuando un recurso se solicita en el player IMS$\mathrm{LD}$, se hace una llamada al servicio $\mathrm{CP}$ para conseguir el recurso más apropiado de acuerdo con las necesidades del estudiante. Si está disponible una alternativa adaptada de ese recurso se mostrará éste en lugar del original.

\section{DIFICULTADES ENCONTRADAS EN EL DESARROLLO DEL CURSO}

El player para IMS-LD utilizado para el desarrollo del curso en la plataforma dotLRN, se encuentra actualmente en desarrollo y se han encontrado diversas dificultades en la implementación técnica del mismo a través de esta especificación. De forma específica aparecen algunas limitaciones técnicas a la hora de desarrollar servicios y tareas que deberían ser incluidas en el flujo de aprendizaje y en los recursos de apoyo asociados. Es necesario realizar nuevos desarrollos que permitan la integración de éstos con el modelo de usuario. En el grupo aDeNu se ha trabajado en este sentido a través de la identificación de problemas y el establecimiento de sus posibles soluciones. Entre ellos se han detectado y resuelto las siguientes dificultades:

- Integración del player IMS-LD con el servicio de Modelado de Usuario

- Solución de problemas relacionados con recursos que utilizaban hojas de estilo (recursos dinámicos como cuestionarios, resultados, registro de estudio...). Los recursos comentados anteriormente no se mostraban de forma apropiada apareciendo diversos errores en el navegador (colores y tamaños no adecuados, etc.).

- Establecimiento adecuado del orden de evaluación de las condiciones lógicas, éstas eran evaluadas en el mismo orden en el que fueron establecidas inicialmente por el autor del diseño de aprendizaje, lo que inducía a errores lógicos y efectos indeseados (p.e.se mostraban en el árbol de actividades tareas que deberían permanecer como no visibles, resultados incorrectos de los cuestionarios, etc.). 
E. Campo; M. Saneiro; M. Montecelo; E. Raffenne; A. Rodriguez; O. Santos; J. Boticario Apoyo AdAPTATIVo BASADO EN IMS-LD y ESTRATEGIAS PSICO-EDUCATIVAS PARA LA FAMILIARIZACión...

- Solución de problemas relacionados con la visualización de determinadas secciones en la interfaz, específicamente cuando todos los elementos no habían sido apropiadamente aplicados dentro de la estructura de una actividad, no se mostraban (p.e. no aparecían elementos marcados como encabezamientos o viceversa).

Asimismo, se han detectado problemas de accesibilidad de la interfaz de usuario del player IMS-LD, respecto a la pautas de accesibilidad al contenido web del $\mathrm{W}_{3} \mathrm{C}$ (WCAG1.o y 2.0). Los problemas detectados, así como las mejoras realizadas en el player han sido comunicadas a la comunidad de dotLRN de cara a considerar su inclusión en la próxima versión del software.

\section{CONCLUSIONES Y TRABAJOS FUTUROS}

El grupo aDeNu de UNED trabaja en el desarrollo de entornos de aprendizaje accesibles y adaptativos que consideran las características específicas del estudiante, para que éste pueda alcanzar un grado alto de rendimiento según sus fortalezas y debilidades. Para ello, en el marco del desarrollo de los proyectos EU4ALL y A2UN@, se ha abordado la elaboración de un curso centrado en la familiarización del estudiante con el entorno virtual de aprendizaje, considerando los siguientes temas: 1) Diseño de escenarios de apoyo considerando las necesidades específicas del estudiante, 2) Implementación de estos escenarios como unidades de aprendizaje a través de la especificación IMS-LD, 3) Integración de estas unidades con los servicios de apoyo necesarios en la plataforma dotLRN, 4) Aplicación de dichas adaptaciones al estudiante en un entorno educativo real.

La siguiente fase será la evaluación del impacto del modelo desarrollado. Para ello el plan de evaluación considerará: i) impacto sobre el currículo, ii) proceso de instrucción, iii) rendimiento académico, satisfacción del alumno y iv) proceso de evaluación. Todo ello de acuerdo a las interacciones con el sistema y los recursos ofrecidos (Campo et al, 2010).

\section{REFERENCIAS BIBLIOGRÁFICAS}

Barba, R. (1993). The effects of embedding an instructional map in hypermedia courseware. Journal of Research on Computing in Education, 25 (4), (405412).
Blöchl, M.; Rumetshofer, H.; Wöß, W. (2003). Individualized E-Learning Systems Enabled by a Semantically Determined Adaptation of Learning Fragments. Proceedings of the 14th 
E. Campo; M. Saneiro; M. Montecelo; E. Raffenne; A. Rodriguez; O. Santos; J. Boticario

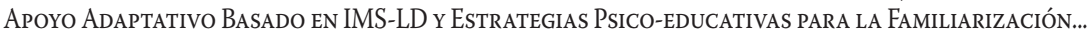

International Workshop on Database and Expert Systems Applications (DEXA'o3). Description of the evaluation scenario

Bloom, B. S. et al. (Eds.) (1984). Taxonomy of Educational Objectives. Book 1: Cognitive Domain. White Plains, NY: Longman

Boulet, A.; Savoie-Zajc, L.; Chevrier, J. (1996). Les stratégies d'apprentissage à l'université. Québec (Canadá): Presses de l'université du Québec

Burgos, D.; Tattersall, C.; Koper, R. (2007). How to represent Adaptation in eLearning with IMS Learning Design. Interactive Learning Environments, 15(2), (161-170).

Campo, E.; Saneiro, M.; Rodríguez, A.; Finat,C.; G. Boticario, J. (2010). Metodología de evaluación de entornos de e-learning, servicios y recursos adaptados a las características de los usuarios. III Jornadas de Redes de Investigación en Innovación Docente. UNED. Madrid.

Carmel, D.; Markovitch, S. (1998). Modelbased learning of Interaction strategies in Multi-Agent System. Journal of Experimental and theorical artificial intelligence, 10, (309-332)

Cercone, K. (2008). Characteristics of adult learners with implications for online learning design. AACE Journal, 16 (2), (137-159).

Ellis, E. S. (1993). Integrative strategy instruction: A potential model for teaching content area subjects to adolescents with learning disabilities. Journal of Learning Disabilities, 26 (6), (358-383).

Escobedo, J. P.; de la Fuente, L.; Gutiérrez, S.; Pardo, A.; Delgado, C. (2007). Implementation of a Learning Design Run-Time Environment for the .LRN Learning Management System. Journal of Interactive Media in Education (Adaptation and IMS Learning Design. Special Issue, ed. Daniel Burgos).
Felder, R. M.; Silverman, L. K. (1988). Learning and Teaching Styles in Engineering Education. Engr. Education, 78(7), 674-681.

Fuente Valentín, L.; De la Pardo, A.; Delgado Kloos, C. (2007). Experiences with GRAIL: Learning Design supportin .LRN. TENCompetence Open workshop on current research in IMS Learning Design and lifelong competence development infrastructures. June 2007.

Harasim, L.; Hiltz, S.; Turoff, M.; Teles, L. (2000). Redes de aprendizaje: Guía para la enseñanza y el aprendizaje en red. Barcelona: Gedisa/EDIUOC (Versión en Inglés Learning networks. A fiel guide to teaching and learning online. Cambridge (EE.UU.): Massachusetts Institute of Technology, 1995)

Hofmeester, G. H. (1996).Sensuality in product design: a structured approach. En: CHI'96 Conference Proceedings (428-435)

IMS (2003). IMS Learning Desing. Retrieved. [en línea] Disponible en: www.imsglobal. org [consulta 2006, 13 de Abril]

Johnson, L. A.; Graham, S.; Harris, K. R. (1997). The effects of goal setting and self-instruction on learning a reading comprehension strategy: A study of students with learning disabilities. Journal of Learning Disabilities, 30(1), (80-91).

Knapper, C. K.; Cropley, A. J. (2000). Lifelong learning in higher education. (3rd ed.). London: Kogan Page.

Koper, R.; Tattersall, C. (Eds.). (2005). Learning Design - A Handbook on Modelling and Delivering Networked Education and Training. Heidelberg: Springer Verlag.

Lerner, J. (1997). Learning disabilities: Theories, diagnosis, and teaching strategies (7th ed.). Boston, MA: Houghton Mifflin.

Logan, R. J. (1994). Behavioral and emotional usability; Thomson consumer electronic. 
E. Campo; M. Saneiro; M. Montecelo; E. Raffenne; A. Rodriguez; O. Santos; J. Boticario Apoyo AdAPTATIVo BASADO EN IMS-LD y ESTRATEGIAS PSICO-EDUCATIVAS PARA LA FAMILIARIZACión...

In: Wiklund, M. E. (Ed.). Usability in practice: how companies develop user friendly products. Academy Press, Boston, (59-82).

Martín Mozón, I. (2007). Estrés académico en estudiantes universitarios. Apuntes de Psicología. Colegio Oficial de Psicología de Andalucía Occidental y Universidad de Sevilla. 2007, Vol. 25, número 1, (8799).

Merrill, D. M. (1997). Instructional Strategies that Teach. CBT Solutions, November/ December, 1-11.

Ridley, D. S.; Schutz, P. A.; Glanz, R. S.; Weinstein, C. E. (1992). Self-regulated learning: the interactive influence of metacognitive awareness and goalsetting. Journal of experimental Education, 60 (4), (293-306).

Santos, O. C.; Boticario, J. G.; Raffenne, E.; Pastor, R. (2007). Why using dotLRN? UNED use cases. FLOSS International Conference.

Swan, K.; Shea, P.; Fredericksen, E.; Pickett, A.; Pelz, W.; Maher, G. (2000). Building knowledge building communities:consistency, contact and communication in virtual classroom. Journal Educational Computing Research, 23(4), (359-381).

Thornburg, D.; Pea, R. (1991). Synthesizing instructional technologies and educational culture: Exploring cognition and metacognition in the social studies. Journal of Educational Computing Research, 7 (2), (121-164).

Van Rosmalen, P.; Boticario, J. (2005). Using Learning Design to support design- and runtime Adaptation. In: Koper, R.; Tattersall, C. (Eds.). Learning Design: A Handbook on Modeling and Delivering Networked Education and Training. Heidelberg, Germany: Springer Verlag.

Whitlock, Q. (2001). Course design for online learning - what's gone wrong. In: Stephenson, J. (Ed.). Teaching Online \& Learning Online: Pedagogies for New Technologies, (182-191). London: Kogan Page Limited.

Wood, E.; Woloshyn, V. E.; Willoughby, T. (1995). Cognitive strategy instruction for middle and high schools. Cambridge, MA: Brookline.

\section{PERFIL ACADÉMICO Y PROFESIONAL DE LOS AUTORES}

Ma Elena del Campo Adrián Profesora Titular de la UNED, trabaja desde hace varias décadas en el tema de la discapacidad, en los diferentes niveles de la vida del sujeto. Coordinadora durante varios años de la Unidad de Atención a la Discapacidad de la UNED. Coordinadora del grupo de investigación de la UNED "Psicobiotecnomedicina en discapacidad y Tercera Edad" (G133E43). Integrante de la Red RETADIM (DPI2005-25234-E) centrada en la investigación de desarrollos tecnológicos aplicados al ámbito de la discapacidad. Autora de numerosas publicaciones y ponencias presentadas a congresos nacionales e internacionales. Representante española de la European Agency for Development in Special Needs Education. Directora de los Cursos de Master, Especialista y Experto Universitario sobre Discapacidades Funcionales, impartido por la UNED. Forma parte del equipo investigador aDeNu de la UNED (Ref:G74E25) en los diversos proyectos que este desarrolla. Revisora de proyectos de investigación del IMSERSO (Instituto 
E. Campo; M. Saneiro; M. Montecelo; E. Raffenne; A. Rodriguez; O. Santos; J. Boticario Apoyo Adaptativo Basado en IMS-LD y Estrategias PSico-EduCATIVAS PARA La FAMILIARIZACión...

de Migració y Servicios Sociales) y la ANEP (Agencia Nacional de Evaluación y Prospectiva).

E-mail: mcampo@psi.uned.es

Ma Del Mar Saneiro Silva Psicólogo clínico especialista en discapacidad. Técnico investigador integrante del equipo de investigación del grupo aDeNu de la UNED (Ref:G74E25). Miembro de la Red RETADIM (Ref:DPI200525234-E) centrada en investigación de desarrollo tecnológicos aplicados al ámbito de la discapacidad. Miembro del grupo de investigación de la UNED Psicobiotecnomedicina en discapacidad y Tercera Edad (Ref:G133E43). Profesortutor de la UNED. Autora de bibliografía relacionada con el tema de la discapacidad. Ha presentado ponencias a congresos nacionales e internacionales sobre la temática. Integrante del equipo docente del Master, Especialista y Experto Europeo sobre Discapacidades Funcionales, impartido por la UNED.

E-mail: masterdiscap@psi.uned.es

Manuel A. Fernández Montecelo Ingeniero Técnico en Informática de Sistemas (UNED; 2005), Ingeniero en Informática a falta de presentar Proyecto de Fin de Carrera (UNED). Trabajos previos en el área de e-Learning (Tec-InFor, UNED; 2000-2001) como becario; en el área de Grid Computing en laboratorio de física de altas energías y partículas (LIP, Lisboa, Portugal; 2006-2009). Seleccionado como participante en 3 ediciones de Google Summer of Code como estudiante (2007, 2008 y 2009). Trabaja como Investigador e Ingeniero de Software en aDeNu desde 2009.

E-mail: mafm@dia.uned.es

Emmanuelle Raffenne Ingeniera de software con experiencia significativa (más de 10 años) en desarrollo técnico y coordinación de desarrollo de aplicaciones enmarcadas en el área de innovación tecnológica. Coordinó durante más de 5 años el grupo Innova, responsable del desarrollo de la plataforma de e-formación de la UNED. Actualmente coordina el desarrollo del grupo de investigación aDeNu de la UNED. Ha participado en varios proyectos de ámbito nacional e internacional y es miembro activo de la comunidad OpenACS (framework para el desarrollo de aplicaciones web).

E-mail: erafenne@dia.uned.es

Alejandro Rodríguez Ascaso Doctor Ingeniero de Telecomunicación por la Universidad Politécnica de Madrid. Actualmente es profesor del Departamento de Inteligencia Artificial de la Universidad Nacional de Educación a Distancia (UNED) e investigador del grupo aDeNu de dicha universidad. Desempeña actividades 
docentes, y de investigación en temas de diseño para todos, TIC como apoyo a la autonomía personal y vida independiente, accesibilidad electrónica y adaptación de recursos de aprendizaje a la diversidad funcional de las personas. Participa en actividades de normalización nacionales e internacionales en AENOR y ETSI. Es miembro del Comité de Expertos TIC de Fundación ONCE y del Centro Nacional de Tecnologías para la Accesibilidad.

Olga C. Santos Directora Técnica de I+D del Grupo de Investigación aDeNu de la UNED. Diploma de Estudios Avanzados en Inteligencia Artificial (UNED, España). Ha participado -a nivel nacional e internacional- en 12 proyectos de investigación, publicado más de 80 artículos en conferencias y revistas y ha sido miembro del comité de programa en más de 25 encuentros científicos, 10 de los cuales también como miembro del comité organizador.

E-mail: ocsantos@dia.uned.es

Jesús González Boticario. Dirige el grupo de investigación aDeNu de la UNED aDeNu. Profesor Titular de Informática de la UNED. Coordinador científico en proyectos nacionales y europeos de investigación en el área de e-inlcusion y e-learning. Ha participado en 18 proyectos de investigación financiados. Organizador de conferencias y workshops en el área de modelado de usuario en sistemas de aprendizaje. Evaluador de proyectos y artículos de revistas y conferencias nacionales e internacionales. Ha tenido diversos cargos en la UNED en el área de la tecnología aplicada a la educación (p.ej., Vicerrector de Innovación y Desarrollo Tecnológico). Coordinador del proyecto de Accesibilidad y Diversidad Funcional dentro de las Redes de Innovación Docente de la UNED. Miembro del Consejo Asesor del Centro de Atención a Universitarios con Discapacidad de la UNED (UNIDIS).

E-mail:jgb@dia.uned.es

DIRECCIÓN DE LOS AUTORES

Grupo de Investigación aDeNu Despacho 3.01 ETS Informática UNED 28040 Madrid

Fecha de recepción del artículo: 15/05/10

Fecha de aceptación del artículo: 27/07/10 